\title{
AN INVERSE KINEMATIC MODEL OF THE HUMAN TRAINING CENTRIFUGE MOTION SIMULATOR
}

\author{
RAFAE LEWKOWICZ \\ Military Institute of Aviation Medicine, Warsaw, Poland \\ e-mail: rlewkowicz@wiml.waw.pl
}

Grzegorz KowALECZKO

Air Force Institute of Technology, Warsaw, Poland

e-mail: grzegorz.kowaleczko@itwl.pl

\begin{abstract}
The paper presents an inverse kinematic model for a centrifuge motion simulator used to verify newly defined absolute acceleration profiles. The modelling is concerned with a human training centrifuge with three degrees of freedom. The values of kinematic parameters have been obtained for this three-jointed manipulator. Validation of the developed model has been performed by comparing the results obtained from the centrifuge motion simulator with the results of numerical simulations. The simulation revealed that the inverse kinematic model enabled calculation of the angular displacement, velocity and acceleration of the links that are needed for the given linear acceleration of the simulator cabin.
\end{abstract}

Keywords: inverse kinematic, simulator, centrifuge, motion system

\section{Introduction}

High-performance aircraft pilots as well as civilian aerobatic pilots are exposed to high linear accelerations during flight (Newman, 2015). In order to properly prepare for work in this environment, pilots are evaluated and trained to increase their acceleration tolerance level (Wojtkowiak, 1991). This training is carried out in a specially designed centrifuge motion simulator or, socalled, human training centrifuge (HTC) (Dančuo et al., 2012b; Truszczyński and Kowalczuk, 2012). From the standpoint of classical mechanics, the task of the HTC is to achieve accelerations through rotations around three axes that simulate the load the pilot is exposed to in a real flight. This type of simulator makes it possible to create high and prolonged linear accelerations. Moreover, the centrifuge provides a safe ground-based platform to train pilots, especially in the field of increasing pilot's awareness about unwanted effects of accelerations, such as G-induced loss of consciousness or spatial disorientation. In addition, the HTC is an important tool for researchers to understand the changes taking place in human physiology during accelerative stress.

In this paper, a dynamic flight simulator has been considered, namely the HTC shown in Fig. 1, manufactured by the AMST-Systemtechnik GmbH (Austria), located at the Military Institute of Aviation Medicine (Warsaw, Poland).

The gondola/cabin of the centrifuge is assembled on an eight-meter-long arm and allowed longitudinal accelerations (in the direction from pilot's head to foot) to be achieved in the range from $-3 g$ to $+16 g$ ( $g$ is the Earth's gravitational acceleration) with the maximal onset rate of acceleration (G-onset rate) at $n=14.5 \mathrm{~g} / \mathrm{s}$. Additionally, the gyroscopic suspension of the cabin allowed it to achieve transversal and lateral accelerations in the range of $\pm 10 \mathrm{~g}$ and $\pm 6 g$, respectively. 


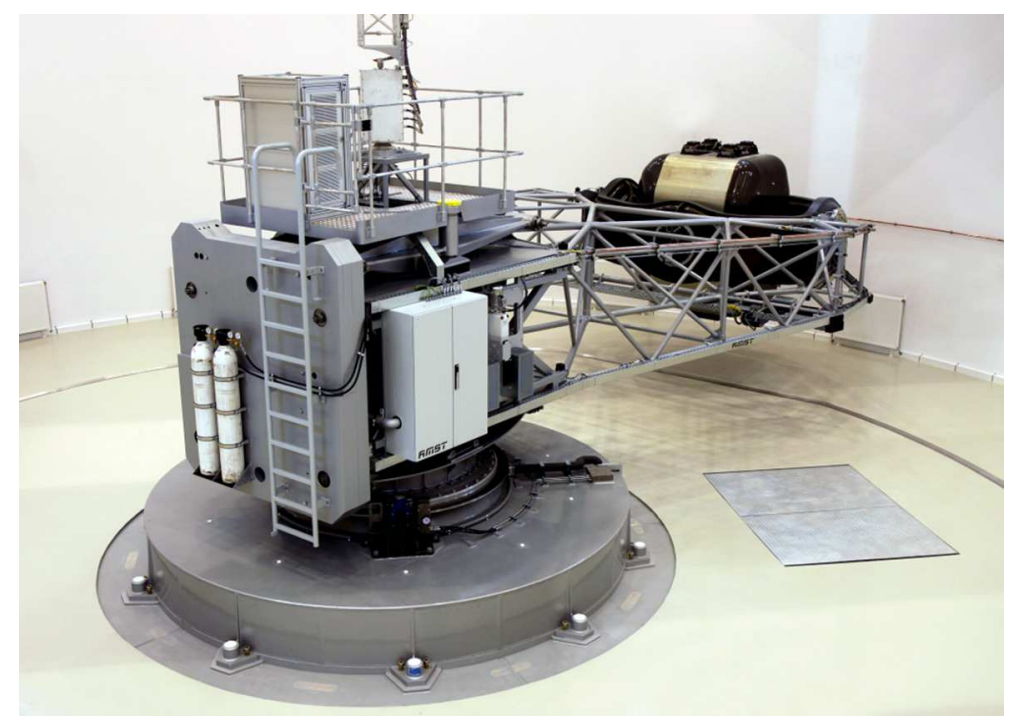

Fig. 1. Dynamic flight simulator - human training centrifuge HTC-07

\section{Problem formulation}

Within the centrifuge simulator, there is a pre-programmed standard open loop mode that contains predefined nonlinear profiles of the absolute acceleration in the centre of the cabin. In these profiles, which are independent for each of the three axes of the pilot's head-fixed coordinate system, a positive or negative acceleration that is constant for given periods of time is determined. When this acceleration profile is created, the angular accelerations in the individual links of the simulator motion system may be exceeded (Table 1). There is also a problem concerning hypogravity $(<1 g)$, which cannot be obtained in this simulator. In that case, the simulator software reports an error, which is then eliminated using a trial and error method. This problem makes it difficult to define more complex acceleration profiles that should not be tested on a real device. The solution to this problem should be sought in the inverse kinematics of the HTC's motion system. The inverse kinematics of the centrifuge motion simulator will be based on calculating the angular position, velocity and acceleration of each motion system link. This approach will make it possible to indicate maximum values of angular acceleration that are necessary to achieve a given linear acceleration of the simulator cabin. In this way, how the position of individual links of the motion system should change over time in order to achieve the desired movement of the cabin will be determined.

\section{Physical model of the HTC simulator}

A centrifuge motion simulator is modelled as a three-joint manipulator (Fig. 2) with rotational axes, where the pilot's head is considered to be the end-effector (Crosbie, 1988). The model consists of three links: arm, ring and cabin (Fig. 2).

The arm rotation around the vertical axis is the main motion that achieves the desired acceleration force. The arm carries a gimballed cabin system with two rotational axes providing pitch and roll capabilities. The task of the roll and pitch axes is to direct the acceleration force into the desired direction. The pilot's head is placed in the intersection of the cabin roll and pitch axes. The arm rotation angle is denoted by $\psi_{A}$, the roll ring rotation angle by $\varphi_{R}$ and the pitch cabin rotation angle by $\theta_{C}$. The centrifuge has the following parameters: arm length $d_{A}=8 \mathrm{~m}$, roll and pitch axis rotation range of $\pm 360^{\circ}$. Other parameters of the simulator motion system kinematics are shown in Table 1. 


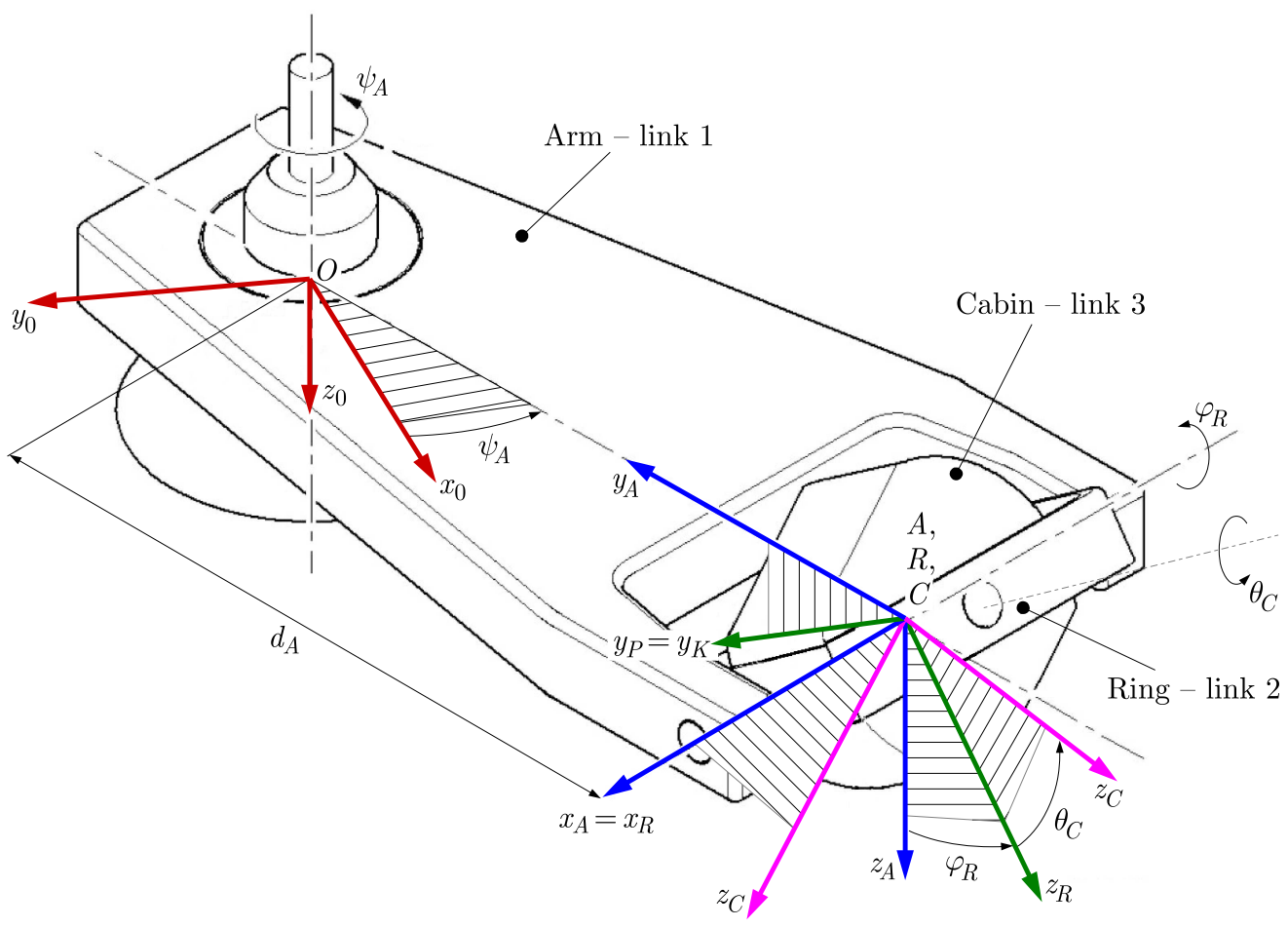

Fig. 2. A physical model of the centrifuge motion simulator HTC-07

Table 1. Motion capabilities of the HTC-07 simulator (AMST-Systemtechnik GmbH, 2011)

\begin{tabular}{|l|c|c|c|}
\hline \multicolumn{1}{|c|}{ Parameter } & $z_{c}$-axis & $y_{c}$-axis & $x_{c^{-}}$axis \\
\hline \hline Maximum acceleration rate $[\mathrm{g} / \mathrm{s}]$ & 14.5 & 6 & 10 \\
\hline Maximum angular acceleration $\left[\mathrm{rad} / \mathrm{s}^{2}\right]$ & 2.82 & 8 & 5 \\
\hline
\end{tabular}

\section{Kinematics of the centrifuge motion simulator}

The forward kinematics that is related to the simulator motion system geometry is used to calculate the linear acceleration components of the end-effector (the pilot's head), where $\mathbf{G}_{H}=\left[G_{x_{H}}, G_{y_{H}}, G_{z_{H}}\right]^{\mathrm{T}}$ with respect to the centrifuge variables $\psi_{A}, \phi_{R}$ and $\theta_{C}$ (these angles are determined in the next Section). Thus, for a given joint coordinate vector $\mathbf{q}=\left[\ddot{\psi}_{A}, \dot{\psi}_{A}, \theta_{C}, \ddot{\theta}_{C}, \phi_{R}, \ddot{\phi}_{R}\right]^{\mathrm{T}}$, the forward kinematics equation must be solved as follows

$$
\mathbf{G}_{H}=f(\mathbf{q})
$$

where $f$ is a nonlinear, continuous and differentiable function. The simulator kinematic model can be derived by different methods, such as the Lagrange equation (Siciliano et al., 2009; Wu et al., 2010), the Newton-Euler method (Grotjahn et al., 2004; Tsai, 1999) and the virtual work principle (Wu et al., 2009, 2013; Zhao and Gao, 2009). The method based on the Lagrange formulation is conceptually simple and systematic. The method based on the Newton-Euler formulation yields a model in a recursive form. It is composed of forward computation of velocities and accelerations of each link, followed by backward computation of forces and moments in each joint (Wu et al., 2010). This algorithm is computationally more efficient because it exploits the typically open structure of the manipulator kinematic chain (Djuric et al., 2012; Siciliano et al., 2009). On the other hand, the Newton-Euler procedure is very difficult to use in an advanced control application because of the closed structure, as the expense of calculation is considerably high (Gherman et al., 2012). Despite this, the Newton-Euler equations of motion 
are used to model the kinematics of centrifuge motion simulator due to the fact that these equations incorporate all accelerations that act on the individual links of the motion system (Chen and Repperger, 1996; Dančuo et al., 2012a; Kvrgic et al., 2014; Vidaković et al., 2012). During kinematic modelling of the centrifuge, the small elastic deformation of the centrifuge links is neglected.

\subsection{Coordinate frames and matrices determining relations for centrifuge links}

This Section defines coordinate frames for the centrifuge links (Fig. 2) and matrices that determine their relations. The centrifuge links and their coordinate frames are denoted by using the Euler angle convention. The centrifuge base coordinates are denoted by $O x_{0} y_{0} z_{0}$ (the Earth-fixed system), the arm coordinates by $A x_{A} y_{A} z_{A}$ (link 1), the roll ring coordinates by $R x_{R} y_{R} z_{R}$ (link 2), the cabin coordinates by $C x_{C} y_{C} z_{C}$ (link 3) and the pilot head-fixed coordinates system by $H x_{H} y_{H} z_{H}$. The pilot's head is placed in the intersection of the cabin roll and pitch axes, therefore $x_{C}=x_{H}, y_{C}=y_{H}$, and $z_{C}=z_{H}$. To determine the mutual position of the defined coordinate systems, the following angles are used (Fig. 2):

- $\psi_{A}$ - the yaw angle between the $x_{0}$-axis and the $y_{A}$-axis. This angle, enlarged by $90^{\circ}$, provides coverage of the $y_{0}$-axis with the $y_{A}$-axis, in this way defining the position of the Earth-fixed coordinate system $O x_{0} y_{0} z_{0}$ relative to the arm-fixed coordinate system $A x_{A} y_{A} z_{A}(\operatorname{link} 1)$

- $\phi_{R}$ - the roll angle between the $z_{A}$-axis and the $z_{P}$-axis. This angle determines the position of the arm-fixed coordinate system $A x_{A} y_{A} z_{A}$ (link 1) relative to the ring-fixed coordinate system $R x_{R} y_{R} z_{R}$ (link 2),

- $\theta_{C}$ - the pitch angle between the $z_{P}$-axis and the $z_{C}$-axis. This angle determines the position of the ring-fixed coordinate system $R x_{R} y_{R} z_{R}$ (link 2) relative to the cabin-fixed coordinate system $C x_{C} y_{C} z_{C}(\operatorname{link} 3)$.

To derive the kinematic equations for the motion simulator, the matrices for the relation between the centrifuge link coordinate frames are defined. These transformation matrices are obtained for the $Z \rightarrow Y \rightarrow X$ rotation convention of the coordinate systems in the following way:

- $\mathbf{L}_{0 / A}$ - matrix for transformation from the Earth-fixed coordinate system $O x_{0} y_{0} z_{0}$ to the arm-fixed coordinate system $A x_{A} y_{A} z_{A}$

$$
\mathbf{L}_{0 / A}=\mathbf{L}_{z_{r}}\left(90^{\circ}\right) \mathbf{L}_{z_{0}}\left(-\psi_{A}\right)
$$

where

$$
\mathbf{L}_{z_{0}}\left(-\psi_{A}\right)=\left[\begin{array}{ccc}
\cos \psi_{A} & -\sin \psi_{A} & 0 \\
\sin \psi_{A} & \cos \psi_{A} & 0 \\
0 & 0 & 1
\end{array}\right] \quad \mathbf{L}_{z_{r}}\left(90^{\circ}\right)=\left[\begin{array}{ccc}
0 & -1 & 0 \\
1 & 0 & 0 \\
0 & 0 & 1
\end{array}\right]
$$

- $\mathbf{L}_{x_{r}}\left(\phi_{R}\right)$ - matrix for transformation from the arm-fixed coordinate system $A x_{A} y_{A} z_{A}$ to the ring-fixed coordinate system $R x_{R} y_{R} z_{R}$

$$
\mathbf{L}_{x_{r}}\left(\phi_{R}\right)=\left[\begin{array}{ccc}
1 & 0 & 0 \\
0 & \cos \phi_{R} & \sin \phi_{R} \\
0 & -\sin \phi_{R} & \cos \phi_{R}
\end{array}\right]
$$

- $\mathbf{L}_{y_{p}}\left(\theta_{C}\right)$ - matrix for transformation from the ring-fixed coordinate system $R x_{R} y_{R} z_{R}$ to the cabin-fixed coordinate system $C x_{C} y_{C} z_{C}$

$$
\mathbf{L}_{y_{p}}\left(\theta_{C}\right)=\left[\begin{array}{ccc}
\cos \theta_{C} & 0 & -\sin \theta_{C} \\
0 & 1 & 0 \\
\sin \theta_{C} & 0 & \cos \theta_{C}
\end{array}\right]
$$


The matrix $\mathbf{L}_{0 / C}$ for transformation from the cabin-fixed coordinate system, $C x_{C} y_{C} z_{C}$, to the Earth-fixed coordinate system, $O x_{0} y_{0} z_{0}$, is determined by multiplying transformation matrices (4.2)-(4.5), in the following way

$$
\mathbf{L}_{0 / C}=\mathbf{L}_{y_{p}}\left(\theta_{C}\right) \mathbf{L}_{x_{r}}\left(\phi_{R}\right) \mathbf{L}_{0 / A}
$$

By using the convenient shorthand notation $c=\cos$ and $s=\sin$, the components of the transformation matrix (4.6) $L_{0 / A}=\mathbf{L}_{z_{r}}\left(90^{\circ}\right) \mathbf{L}_{z_{0}}\left(-\psi_{A}\right)$ (4.2) become the following

$$
\mathbf{L}_{0 / C}=\left[\begin{array}{ccc}
s \psi_{A} c \theta_{C}+c \psi_{A} s \phi_{R} s \theta_{C} & c \psi_{A} c \phi_{R} & -s \psi_{A} s \theta_{C}+c \psi_{A} s \phi_{R} c \theta_{C} \\
-c \psi_{A} c \theta_{C}+s \psi_{A} s \phi_{R} s \theta_{C} & s \psi_{A} c \phi_{R} & c \psi_{A} s \theta_{C}+s \psi_{A} s \phi_{R} c \theta_{C} \\
c \phi_{R} s \theta_{C} & -s \phi_{R} & c \phi_{R} c \theta_{C}
\end{array}\right]
$$

Assuming that $\psi_{A}=0$, for further calculations matrix (4.7) is reduced to the form

$$
\mathbf{L}_{0 / C\left(\psi_{A}=0\right)}=\left[\begin{array}{ccc}
\sin \phi_{R} \sin \theta_{C} & \cos \phi_{R} & \sin \phi_{R} \cos \theta_{C} \\
-\cos \theta_{C} & 0 & \sin \theta_{C} \\
\cos \phi_{R} \sin \theta_{C} & -\sin \phi_{R} & \cos \phi_{R} \cos \theta_{C}
\end{array}\right]
$$

On the basis of these transformational matrices, the equations of forward kinematics that relate to the velocities and accelerations of the links and the end-effector-pilot's head are developed in the next Section.

\section{Linear acceleration acting on the pilot's head}

The linear acceleration components at the intersection point of the roll (link 2) and pitch (link 3$)$ axes are: the normal (radial) $a_{n}$, tangential $a_{t}$ and gravitational $g$ accelerations (Fig. 3). From these accelerations, the orthogonal components $G_{x 0}, G_{y 0}$, and $G_{z 0}$ for the normal, tangential and vertical accelerations, respectively, are calculated as follows

$$
\left[\begin{array}{c}
G_{x 0} \\
G_{y 0} \\
G_{z 0}
\end{array}\right]=\frac{1}{g}\left[\begin{array}{c}
-a_{n} \\
-a_{t} \\
g
\end{array}\right]=\left[\begin{array}{c}
d_{A} \dot{\psi}_{A}^{2} / g \\
-d_{A} \ddot{\psi}_{A} / g \\
1
\end{array}\right]
$$

where $d_{A}$ is the simulator arm length, $\dot{\psi}_{A}$ and $\ddot{\psi}_{A}$ are angular velocity and acceleration of the arm (link 1), respectively.

The link angles $\phi_{R}$ and $\theta_{C}$, angular velocity $\dot{\psi}_{A}$ and acceleration $\ddot{\psi}_{A}$ of the arm define the orthogonal components $G_{x_{C}}, G_{y_{C}}$ and $G_{z_{C}}$ of the resultant vector $\mathbf{G}_{C}$ that are experienced by the pilot. Based on equations (4.8) and (5.1), the resultant vector $\mathbf{G}_{C}$ experienced by the pilot can be found from

$$
\left[G_{x_{C}}, G_{y_{C}}, G_{z_{C}}\right]^{\mathrm{T}}=\mathbf{L}_{0 / C\left(\psi_{A}=0\right)}^{-1}\left[G_{x 0}, G_{y 0}, G_{z 0}\right]^{\mathrm{T}}
$$

The transverse $G_{x_{C}}$, lateral $G_{y_{C}}$ and longitudinal $G_{z_{C}}$ components of the acceleration $\mathbf{G}_{C}$ that act on the pilot's head are

$$
\begin{aligned}
& G_{x C}=\sin \theta_{C}\left(G_{x 0} \sin \phi_{R}+G_{z 0} \cos \phi_{R}\right)-G_{y 0} \cos \theta_{C} \\
& G_{y C}=G_{x 0} \cos \phi_{R}-G_{z 0} \sin \phi_{R} \\
& G_{z C}=\cos \theta_{C}\left(G_{x 0} \sin \phi_{R}+G_{z 0} \cos \phi_{R}\right)+G_{y 0} \sin \theta_{C}
\end{aligned}
$$

Equations (5.3) develop the inverse kinematics algorithm which determines the link angles that are required to generate a desired trajectory of the cabin-centrifuge and accelerations in the centrifuge axes. 


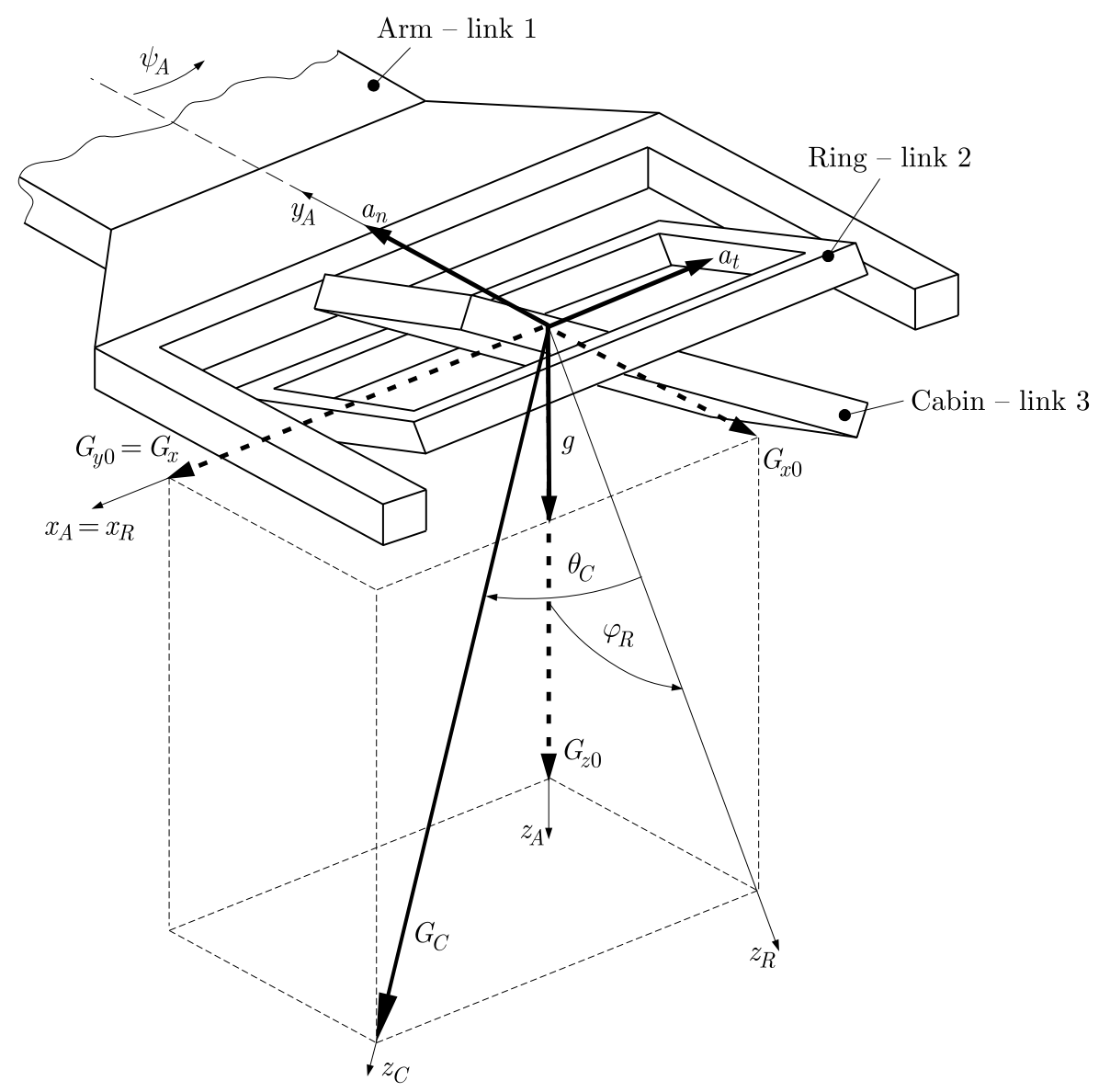

Fig. 3. The acceleration components at the intersection point of the roll and pitch axes

\section{An inverse kinematics of the cabin simulator}

The inverse kinematics is defined as the problem of determining a set of appropriate joint configurations for which the end effector (the pilot's head) moves to desired positions as smoothly, rapidly and as accurately as possible. The inverse kinematics of the centrifuge motion system is first based on calculating the angular displacement, velocity and acceleration of the links that are needed for the given linear acceleration of the simulator cabin. Then, taking into account the limitations of the motion system (Table 1), it is checked whether this system can achieve such accelerations. If it cannot, the maximum successive link angular accelerations that the motion system can achieve are calculated. The inverse kinematics for the centrifuge motion simulator can be described by the relationship

$$
\left[\ddot{\psi}_{A}, \dot{\psi}_{A}, \theta_{C}, \ddot{\theta}_{C}, \phi_{R}, \ddot{\phi}_{R}\right]^{\mathrm{T}}=f^{-1}\left[G_{x_{H}}, G_{y_{H}}, G_{z_{H}}\right]^{\mathrm{T}}
$$

where $f^{-1}$ is a nonlinear, continuous and differentiable function that performs the inverse transformation to the function $f$, (4.1). There are two distinct methods for solving Eq. (6.1) of inverse kinematics, namely iterative and analytical. The iterative method gives the solution by solving an approximation of the system, and by updating the system with the output from the solver for each iteration until it converges. The analytical method solves the whole system at once; however, the complexity of it arises when large chains of joints attempt to be solved. The most prominent among iterative methods are based on the Jacobian matrix, which describes the nonlinear and configuration dependent transformation between velocities in the joint configuration coordinates and the task spaces. There are several versions of Jacobian-based methods, such as 
the Jacobian Transpose (Wolovich and Elliott, 1984), damped least squares (Wampler, 1986), damped least squares with singular value decomposition (Wampler, 1986), selectively damped least squares (Buss and Kim, 2005) and several extensions (Baillieul, 1985; Nakamura and Hanafusa, 1986). An iterative method can be also viewed as an optimization task solved with general-purpose methods (neural networks (Tejomurtula and Kak, 1999) and genetic algorithms (Nearchou, 1998)), but those approaches are usually computationally ineffective.

Due to small chains of joints (a centrifuge simulator is a three-joint manipulator), direct and analytical computations of inverse kinematics are chosen. Based on the acceleration vector $\mathbf{G}_{C}$ components, Eqs. (5.3), the link angles in each joint in the system are derived. To determine the angular accelerations and velocities of the arm (link 1), ring (link 2) and cabin (link 3), the following calculation algorithm for the inverse kinematics is used (Kvrgic et al., 2014).

Step 1: Determination of the arm angular acceleration $\dot{\psi}_{A}$. The angular acceleration $\dot{\psi}_{A}$ is derived from Eq. (5.1) that describes linear acceleration components at the intersection point of the roll and pitch axes (Fig. 3). The resultant acceleration of this point is a sum of the normal $a_{n}$, tangential $a_{t}$, and gravitational $g$ acceleration. This acceleration is as follows

$$
a_{A}^{2}=d_{A}^{2} \dot{\psi}_{A}^{4}+d_{A}^{2} \ddot{\psi}_{A}^{2}+g^{2}
$$

For a positive angular acceleration $\ddot{\psi}_{A}$, the angular velocity $\dot{\psi}_{A}$ in the $i$-th moment of time is equal to

$$
\dot{\psi}_{A}(i)=\dot{\psi}_{A}(i-1)+\ddot{\psi}_{A}(i) d t
$$

By substituting (6.3) to equation (6.2), the resultant acceleration takes the form

$$
a_{A}^{2}(i)=d_{A}^{2}\left[\dot{\psi}_{A}(i-1)+\ddot{\psi}_{A}(i) d t\right]^{4}+d_{A}^{2} \ddot{\psi}_{A}^{2}(i)+g^{2}
$$

After calculations have been performed, this equation becomes

$$
a_{A}^{2}(i)=d_{A}^{2}\left[\dot{\psi}_{A}^{4}(i-1)+4 \dot{\psi}_{A}^{3}(i-1) \ddot{\psi}_{A}(i) d t+6 \dot{\psi}_{A}^{2}(i-1) \ddot{\psi}_{A}^{2}(i) d t^{2}\right]+d_{A}^{2} \ddot{\psi}_{A}^{2}(i)+g^{2}
$$

and then

$$
\frac{a_{A}^{2}(i)-g^{2}}{d_{A}^{2}}=\dot{\psi}_{A}^{4}(i-1)+4 \dot{\psi}_{A}^{3}(i-1) \ddot{\psi}_{A}(i) d t+6 \dot{\psi}_{A}^{2}(i-1) \ddot{\psi}_{A}^{2}(i) d t^{2}+\ddot{\psi}_{A}^{2}(i)
$$

By reducing equation (6.6) to the form of a quadratic equation

$$
\left[1+6 \dot{\psi}_{A}^{2}(i-1) d t^{2}\right] \ddot{\psi}_{A}^{2}(i)+4 \dot{\psi}_{A}^{3}(i-1) \ddot{\psi}_{A}(i) d t+\dot{\psi}_{A}^{4}(i-1)-\frac{a_{A}^{2}(i)-g^{2}}{d_{A}^{2}}=0
$$

it is possible to obtain its solution in the form of two roots

$$
\ddot{\psi}_{A}(i)=\frac{-2 \dot{\psi}_{A}^{3}(i-1) d t \pm \sqrt{-2 \dot{\psi}_{A}^{6}(i-1) d t^{2}-\dot{\psi}_{A}^{4}(i-1)+\left[1+6 \dot{\psi}_{A}^{2}(i-1) d t^{2}\right] k(i)}}{1+6 \dot{\psi}_{A}^{2}(i-1) d t^{2}}
$$

where

$$
k(i)=\frac{a_{A}^{2}(i)-g^{2}}{d_{A}^{2}}
$$

Kvrgic et al. (2014) noted that equation (6.8) is valid for the movement that has a positive acceleration onset. For a negative acceleration onset, the discriminant $-2 \dot{\psi}_{A}^{6}(i-1) d t^{2}-\dot{\psi}_{A}^{4}(i-$ $1)+\left[1+6 \dot{\psi}_{A}^{2}(i-1) d t^{2}\right] k(i)$ is mostly negative, which means that this equation cannot be used 
directly. Vidaković et al. $(2012,2013)$ proposed a solution in the form of a Jacobi elliptic function, which describes the arm angular velocity as

$$
\dot{\psi}_{A}(t)=\sqrt[4]{k} \operatorname{sn}\left(\sqrt[4]{k} t+\sqrt[4]{k} C_{1},-1\right)
$$

where $k$ is constant for every interpolation period of time and is given by Eq. (6.9), sn is a Jacobian elliptic function and $C_{1}$ is the constant obtained from the value of angular velocity from the previous interpolation period.

After equation (6.10) has been developed in Taylor series expansions of the Jacobi elliptic function (Wrigge, 1981), it becomes

$$
\dot{\psi}_{A}(i)=\sqrt[4]{k(i)}\left(t_{1}(i)-\frac{t_{1}^{5}(i)}{10}+\frac{t_{1}^{9}(i)}{120}-\frac{11 t_{1}^{13}(i)}{15600}+\frac{211 t_{1}^{17}(i)}{3536000}\right)
$$

where $t_{1}(i)=\sqrt[4]{k(i)}\left(d t+C_{1}\right)$.

Equation (6.11) describes the arm angular velocity, $\dot{\psi}_{A}(i)$, for each $i$-th interpolation period of time. The angular acceleration, $\ddot{\psi}_{A}(i)$, of the arm for every interpolation period of time is calculated as

$$
\ddot{\psi}_{A}(i)=\frac{\dot{\psi}_{A}(i+1)-\operatorname{dot} \psi_{A}(i)}{d t}
$$

Another approach to solve the problem of calculation of the negative acceleration was proposed by Liwen et al. (2015). The researchers generated both a trapezoidal G-load curve and three-axis G-load commands using a real-time motion planning algorithm with two G-dimensional interpolation. Dančuo et al. (2018) and Vidaković et al. (2012) indicated that equation (6.8) could be also solved numerically for a small time increment $d t \rightarrow 0$.

Step 2: Determination of the angular velocity $\dot{\psi}_{A}$ of the arm (6.3) and accelerations components (5.1) at the intersection point of the roll and pitch axes (Fig. 3).

Step 3: Determination of the roll ring angle $\phi_{R}$ based on equation $(5.3)_{2}$, which describes the lateral acceleration $G_{y C}$. Expressing $\sin \phi_{R}$ and $\cos \phi_{R}$ by using the tangent function

$$
\cos \phi_{R}=\frac{1}{\sqrt{1+\tan ^{2} \phi_{R}}} \quad \sin \phi_{R}=\frac{\tan \phi_{R}}{\sqrt{1+\tan ^{2} \phi_{R}}}
$$

and by substituting these functions to equation $(5.3)_{2}$, multiplying both sides by $\sqrt{1+\tan ^{2} \phi_{R}}$, and then raising to the power, the following expression is obtained

$$
G_{x 0}^{2}-2 G_{x 0} G_{z 0} \tan \phi_{R}+G_{z 0}^{2} \tan ^{2} \phi_{R}=G_{y_{C}}^{2}\left(1+\tan ^{2} \phi_{R}\right)
$$

After the next transformation, Eq. (6.14) is reduced to the form

$$
\left(G_{z 0}^{2}-G_{y_{C}}^{2}\right) \tan ^{2} \phi_{R}-2 G_{x 0} G_{z 0} \tan \phi_{R}+G_{x 0}^{2}-G_{y_{C}}^{2}=0
$$

for which the roots are the following

$$
\tan \phi_{R}=\frac{2 G_{x 0} \pm \sqrt{4 G_{x 0}^{2}-4\left(G_{z 0}^{2}-G_{y_{C}}^{2}\right)\left(G_{x 0}^{2}-G_{y_{C}}^{2}\right)}}{2\left(G_{z 0}^{2}-G_{y_{C}}^{2}\right)}
$$

By substituting $G_{z 0}=1$ to Eq. (6.16), and then performing manipulations, the following result is obtained

$$
\tan \phi_{R}=\frac{G_{x 0} \pm G_{y_{C}} \sqrt{1+G_{x 0}^{2}-G_{y_{C}}^{2}}}{1-G_{y_{C}}^{2}}
$$


For $G_{x 0}^{2}+1 \geqslant G_{y_{C}}^{2}$, the roll ring angle is equal to

$$
\phi_{R}=\arctan \frac{G_{x 0}+G_{y_{C}} \sqrt{1-G_{y_{C}}^{2}+G_{x 0}^{2}}}{1-G_{y_{C}}^{2}}
$$

otherwise

$$
\phi_{R}=\arctan \frac{G_{x 0}}{1-G_{y_{C}}^{2}}
$$

If $G_{y_{C}}<0$, and $G_{y_{C}}^{2}>1$, the roll ring angle is equal to $\phi_{R}=\phi_{R}+\pi$. The angular velocity $\dot{\phi}_{R}$ and acceleration $\ddot{\phi}_{R}$ of the ring (link 2) are determined as follows

$$
\dot{\phi}_{R}(i)=\frac{\phi_{R}(i)-\phi_{R}(i-1)}{d t} \quad \ddot{\phi}_{R}(i)=\frac{\dot{\phi}_{R}(i)-\dot{\phi}_{R}(i-1)}{d t}
$$

Step 4: Completion of the pitch cabin angle $\theta_{C}$ calculation. This angle can be derived from equation (5.3) $)_{1}$ that describes the lateral acceleration $G_{x C}$ or based on Eq. (5.3) 3 which defines the longitudinal acceleration $G_{z C}$. Equations $(5.3)_{1}$ and $(5.3)_{3}$ indicate that it is not possible to obtain simultaneously the desired values of $G_{x C}$ and $G_{z C}$ acceleration, even if they do not exceed the limit ranges (Table 1). Therefore, to determine the pitch cabin angle, Eq. $(5.3)_{1}$ is used. For the known lateral acceleration $G_{x C}$, using similar substitution (6.13) for the pitch cabin angle $\theta_{C}$, equation $(5.3)_{1}$ takes the form

$$
G_{x_{C}}=\frac{\tan \theta_{C}}{\sqrt{1+\tan ^{2} \theta_{C}}}\left(G_{x 0} \sin \phi_{R}+G_{z 0} \cos \phi_{R}\right)-G_{y 0} \frac{1}{\sqrt{1+\tan ^{2} \theta_{C}}}
$$

Performing analogous transformations (6.14) and (6.15) as for the angle of tilting the ring, the above equation takes the form

$$
\begin{gathered}
G_{x_{C}}^{2}\left(1+\tan ^{2} \theta_{C}\right)=\tan ^{2} \theta_{C}\left(G_{x 0} \sin \phi_{R}+G_{z 0} \cos \phi_{R}\right)^{2} \\
-2 G_{y 0} \tan \theta_{C}\left(G_{x 0} \sin \phi_{R}+G_{z 0} \cos \phi_{R}\right)+G_{y 0}^{2}
\end{gathered}
$$

By substituting $G_{x 0} \sin \phi_{R}+G_{z 0} \cos \phi_{R}=d$, and then performing some manipulations, a quadratic equation is obtained

$$
\left(d^{2}-G_{x_{C}}^{2}\right) \tan ^{2} \theta_{C}-2 G_{y 0} d \tan \theta_{C}+G_{y 0}^{2}+G_{x_{C}}^{2}=0
$$

for which the roots are the following

$$
\tan \theta_{C}=\frac{G_{y 0} d \pm G_{x_{C}} \sqrt{d^{2}-G_{y 0}^{2}-G_{x_{C}}^{2}}}{d^{2}-G_{x_{C}}^{2}}
$$

For $\left(d^{2}+G_{y 0}^{2}\right) \geqslant G_{x_{C}}^{2}$, the pitch cabin angle $\theta_{C}$ is equal to

$$
\theta_{C}=\arctan \frac{G_{y 0} d+G_{x_{C}} \sqrt{d^{2}+G_{y 0}^{2}-G_{x_{C}}^{2}}}{d^{2}-G_{x_{C}}^{2}}
$$

otherwise, if $\left(d^{2}+G_{y 0}^{2}\right)<G_{x_{C}}^{2}$, it is not possible to obtain the desired transverse acceleration $G_{x C}$. Then equation (6.21) takes the form

$$
\theta_{C}=\arctan \frac{G_{y 0}}{d-G_{x_{C}}^{2} / d}
$$


The angular velocity $\dot{\theta}_{C}$ and acceleration of the cabin $\ddot{\theta}_{C}$ (link 3 ) are determined as follows

$$
\dot{\theta}_{C}(i)=\frac{\theta_{C}(i)-\theta_{C}(i-1)}{d t} \quad \ddot{\theta}_{C}(i)=\frac{\dot{\theta}_{C}(i)-\dot{\theta}_{C}(i-1)}{d t}
$$

A centrifuge is capable of simulating all three load components, but simulating a pure $G_{z C}$ profile becomes a problem due to motor limitation. A pure $G_{z C}$ training profile is a profile without the transverse $G_{x C}$ and lateral $G_{y C}$ loads. The transverse load $G_{x C}$ in the centrifuge is a result of the tangential acceleration $a_{t}$ (Fig. 3) and has a large value at the beginning and at the end of planetary arm motion. The greater the tangential acceleration is, the greater is the $G_{x C}$ acceleration. The emergence of the large tangential acceleration $a_{t}$ decreases the angular velocity of the arm motion and has negative effects on the overall centrifuge performance (Dančuo et al., 2013). These effects are minimized by adjusting the pitch cabin angle by $\theta_{C}=\arctan \left(a_{t} / \sqrt{a_{n}^{2}+1}\right)$.

Equations (6.3), (6.8), (6.18), (6.20), (6.25) and (6.27) compose a system of 8 ordinary differential equations that describe an inverse kinematics model of the HTC motion simulator. Based on these equations, the centrifuge kinematic parameters: $\psi_{A}, \dot{\psi}_{A}, \ddot{\psi}_{A}, \phi_{R}, \dot{\phi}_{R}, \ddot{\phi}_{R}, \theta_{C}$, $\dot{\theta}_{C}$, and $\ddot{\theta}_{C}$ are calculated in three phases, according to the algorithm described by Kvrgic et al. (2014).

\section{Verification of the inverse kinematics model}

The presented inverse kinematics model of the HTC motion simulator has been tested using numerical calculations. The simulation was performed for the $G_{x C}, G_{y C}$ and $G_{z C}$ acceleration forces profile (Fig. 5, solid line), which was generated by the software of the HTC control system. This acceleration forces profile changes as follows:

- starting from $1 g$ with G-onset rate $n=0.2 g / \mathrm{s}$ up to the baseline level (1.41g),

- constant baseline level at $1.41 \mathrm{~g}$,

- increase of the acceleration with G-onset rate $n=3 g / \mathrm{s}$ up to $6 g$,

- constant acceleration at $6 g$,

- decrease of the acceleration with G-onset rate $n=-3 g / \mathrm{s}$ up to the baseline level $(1.41 g)$.

Additionally, for calculations the following data, namely $d_{A}=8 \mathrm{~m}, g=9.81 \mathrm{~m} / \mathrm{s}^{2}$, time step $d t=0.005 \mathrm{~s}$ and Matlab/Simulink MathWorks software, have been used. Figures 4-7 present the results of numerical simulations (dotted line) plotted together with the corresponding parameters which were recorded during operation of the HTC simulator (solid line). The figures show:

- angular velocity $\dot{\psi}_{A}$ and acceleration $\ddot{\psi}_{A}$ of the arm (link 1) (Fig. 4),

- $G_{x C}, G_{y C}$, and $G_{z C}$ acceleration forces profile (Fig. 5),

- angle $\phi_{R}$, angular velocity $\dot{\phi}_{R}$ and acceleration $\ddot{\phi}_{R}$ of the roll ring (link 2) (Fig. 6),

- angle $\theta_{C}$, angular velocity $\dot{\theta}_{C}$ and acceleration $\ddot{\theta}_{C}$ of the pitch cabin (link 3) (Fig. 7).

The calculated angular velocity of the arm $\dot{\psi}_{A}$ (dotted line on the upper plot in the Fig. 4), which is responsible for generating the centripetal acceleration $a_{n}$, largely covers the envelope of the angular velocity obtained from the HTC control system (solid line). The difference between the two curves of $\dot{\psi}_{A}$ (Fig. 4, model vs. HTC) is noticeable only during a decrease in the acceleration from $6 g$ to $1.41 g$ with G-onset rate $n=-3 g / \mathrm{s}$ (Fig. 5). The maximum of this difference is approximately $0.2 \mathrm{rad} / \mathrm{s}$. A similar difference was found in the study by Vidaković et al. (2012).

In Fig. 5, the $G_{x_{H}}, G_{y_{H}}$, and $G_{z_{H}}$ components of the absolute acceleration force obtained by equations (5.3) are given. The curves are very close to each other, except for the phase of 

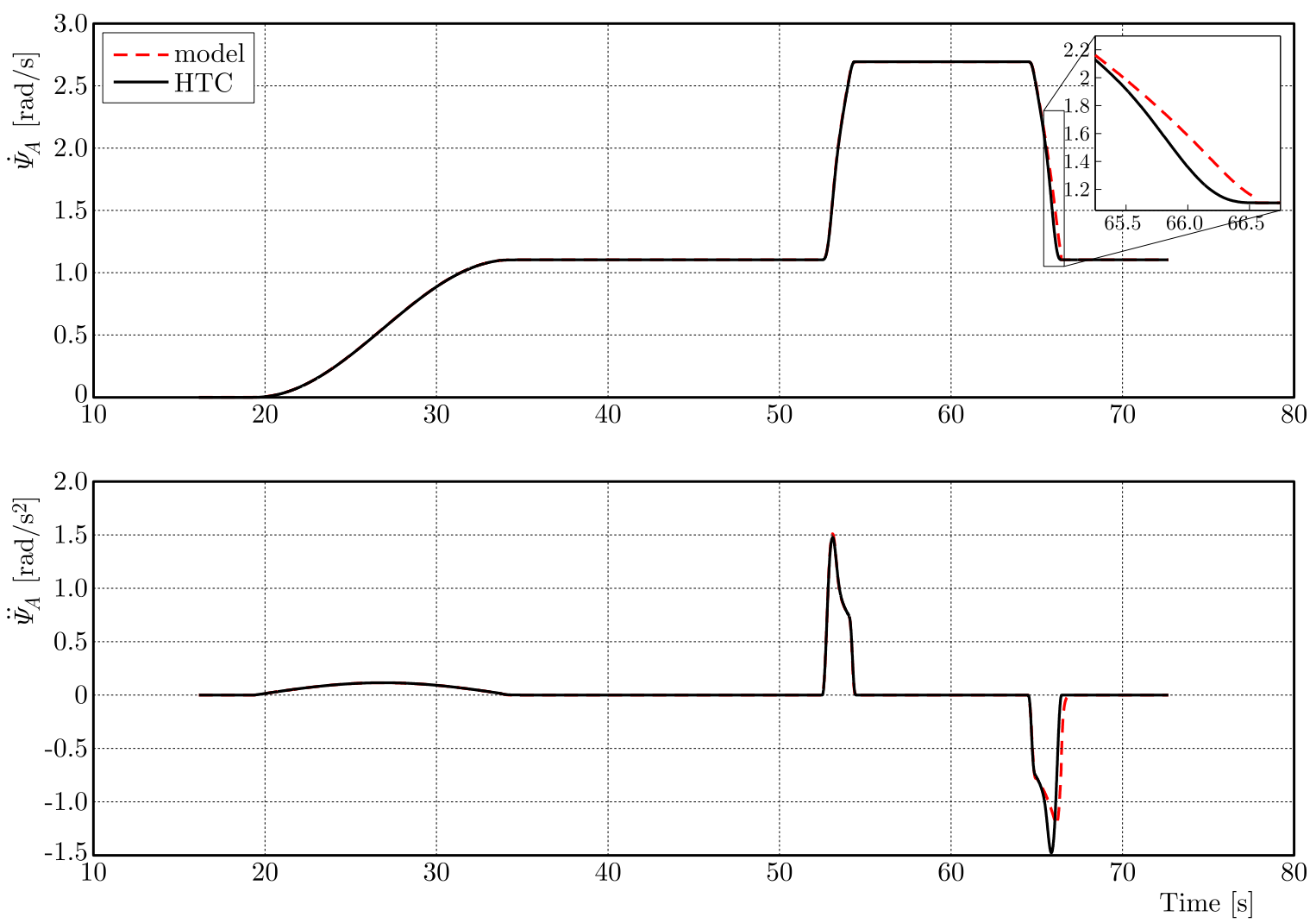

Fig. 4. Angular velocity and acceleration of the centrifuge arm
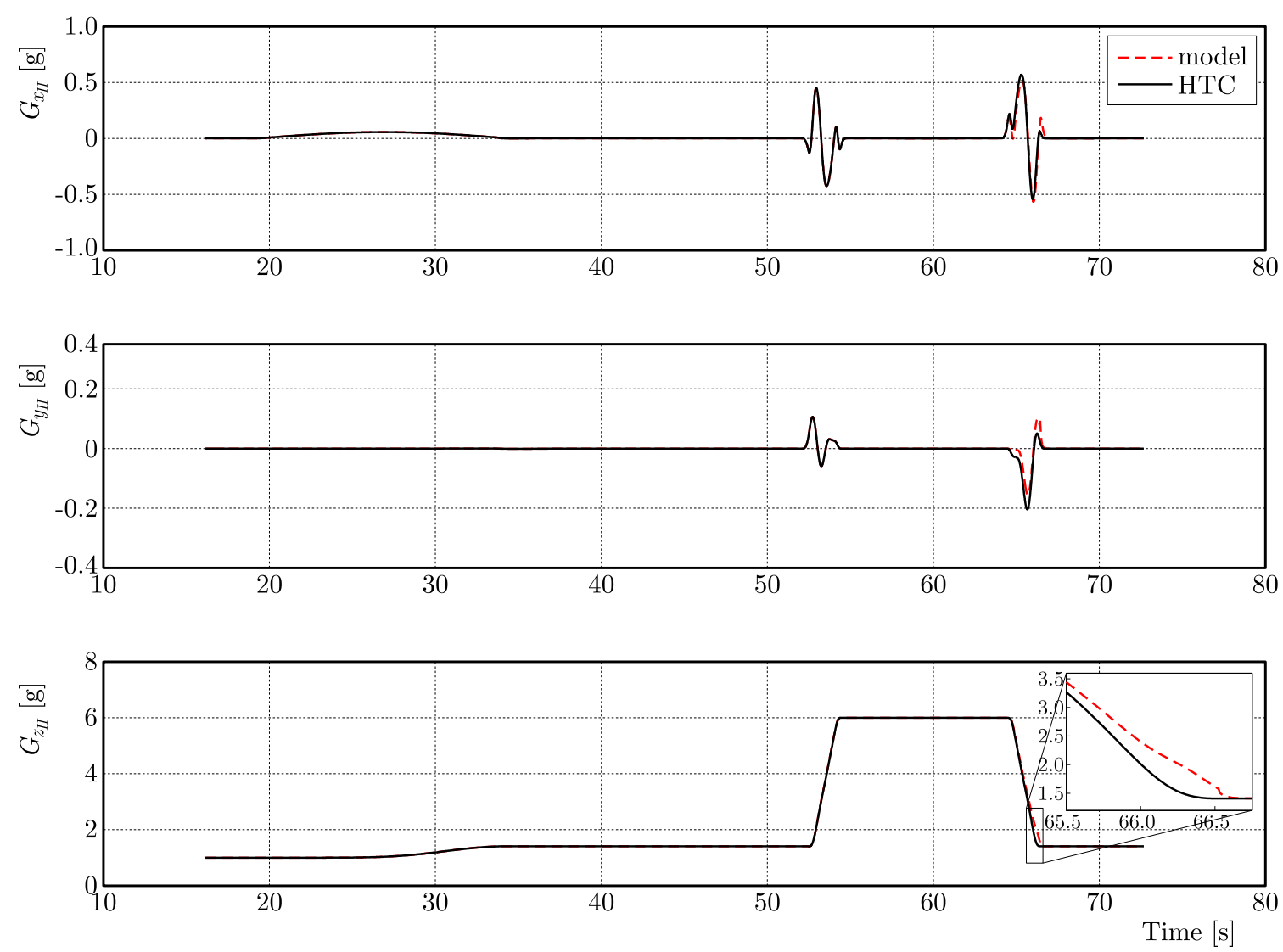

Fig. 5. Components of the absolute acceleration 
deceleration (negative angular acceleration $\ddot{\psi}_{A}$ shown in Fig. 4 ), when a small difference has appeared. The maximum error of the absolute acceleration does not exceed the value of $0.2 g$ $\left(G_{z_{H}}\right)$.

From Fig. 6 , it is clear that the presented angle $\phi_{R}$, angular velocity $\dot{\phi}_{R}$ and acceleration $\ddot{\phi}_{R}$ of the roll ring (link 2) provide good results. A difference between the desired (HTC) and calculated (model) angle $\phi_{R}$ is minimal. The angle $\phi_{R}$ is derived from equation (6.17) and depends on $G_{x 0}$ acceleration (5.1). Thus, the observed difference in the calculated angle $\phi_{R}$ comes from angular velocity of the arm $\dot{\psi}_{A}$ (Fig. 4) which affects $G_{x 0}$ acceleration. Moreover, according to equation (6.20), the angular velocity $\dot{\phi}_{R}$, and acceleration $\ddot{\phi}_{R}$ of the roll ring are calculated on the basis of the angle $\phi_{R}$. Therefore, these parameters curves (model vs. HTC) are also different.
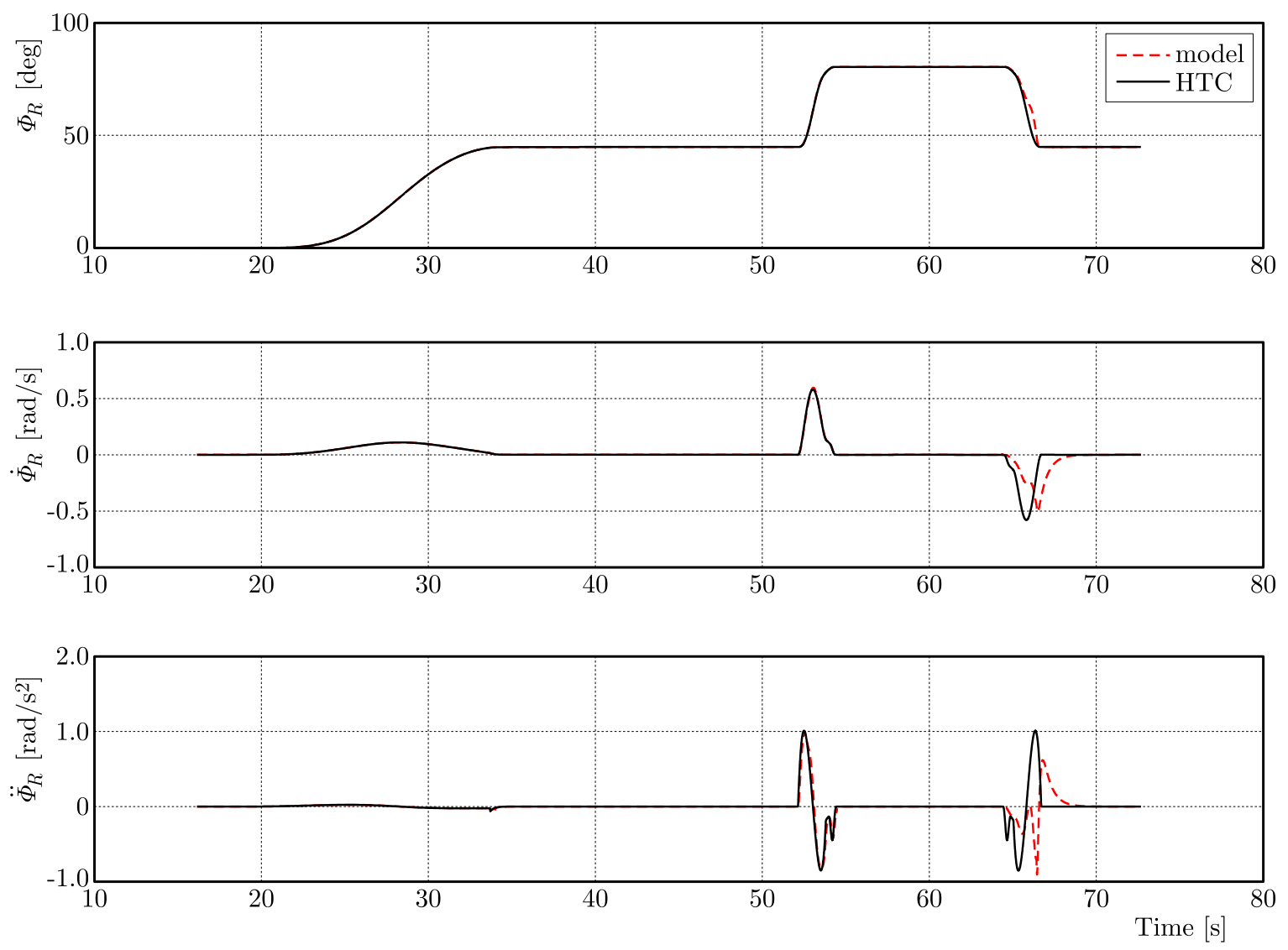

Fig. 6. Kinematic parameters of the roll ring: angle, angular velocity and acceleration

Figure 7 shows the angle $\theta_{C}$, angular velocity $\dot{\theta}_{C}$ and acceleration $\ddot{\theta}_{C}$ of the pitch cabin (link 3) obtained by equations (6.25), and (6.27), respectively. A difference between the desired (solid line) and calculated (dotted line) angle $\theta_{C}$ appears only for the phase of deceleration (negative angular acceleration $\ddot{\psi}_{A}$, shown in Fig. 4) of the arm. Similar to the kinematic parameters calculated for the ring (link 2), the observed difference in the calculated angle $\theta_{C}(6.25)$ depends on the centrifuge arm movement (angular acceleration of the arm $\ddot{\psi}_{A}$, which affects $G_{y 0}$ acceleration (5.1)). The angular velocity $\dot{\theta}_{C}$ and acceleration $\ddot{\theta}_{C}$ of the roll ring (Fig. 7) are calculated based on equation (6.27). Therefore, the differences between two curves (model vs. HTC) of these parameters are easily observed.

The obtained kinematic model is not completely accurate, but the calculated link accelerations, velocities and angles do not differ much from their actual values. It is a concern, especially for maximum and minimum values indicating whether the limit ranges (Table 1) have not been exceeded to achieve the desired values of the acceleration vector $\mathbf{G}_{C}$ components. In order to eliminate the differences between the desired (HTC) and calculated (model) parameters for the 

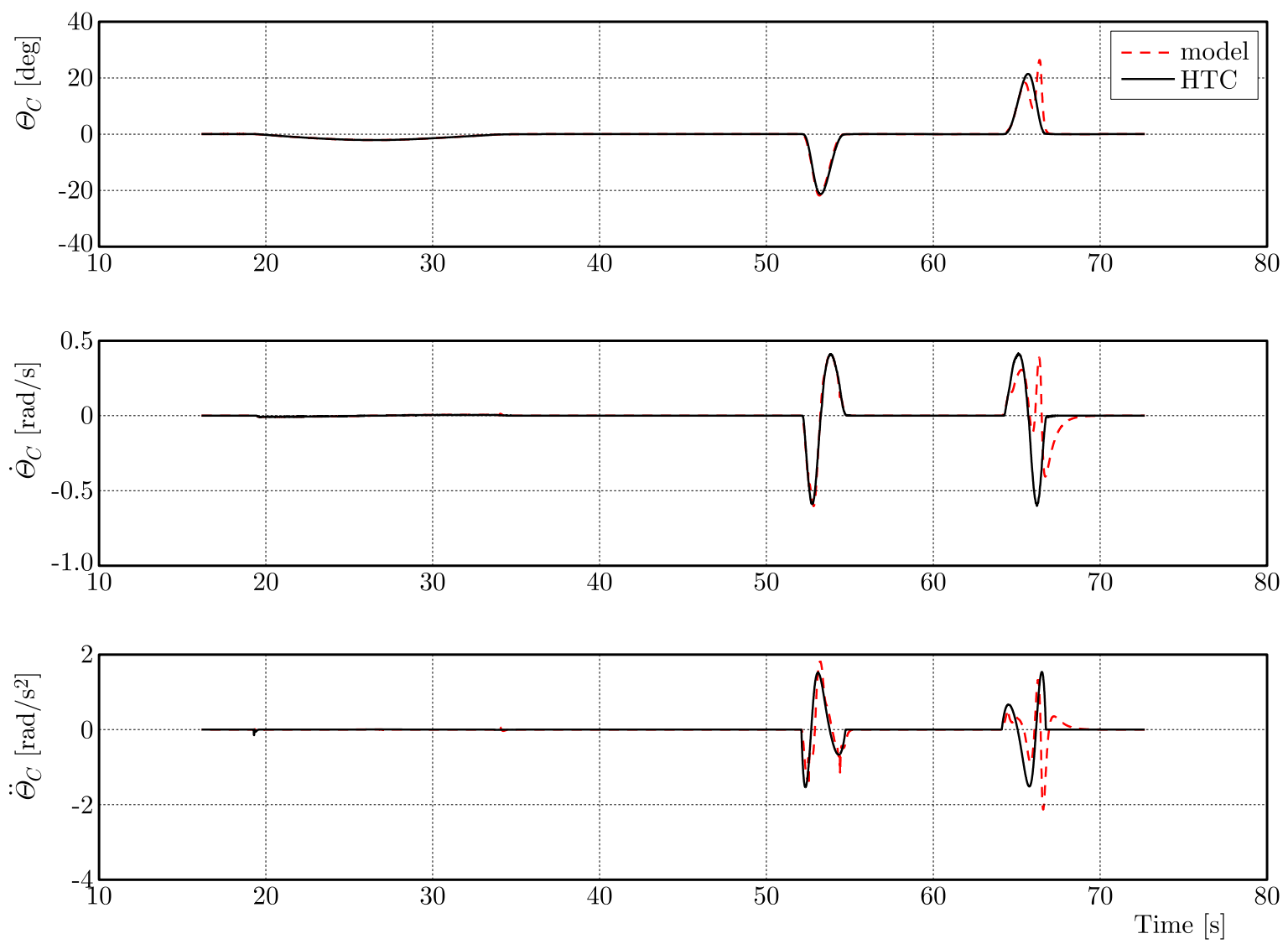

Fig. 7. Kinematic parameters of the pitch cabin: angle, angular velocity and acceleration

movement having a negative acceleration onset, Kvrgic et al. (2014) proposed a simple solution in which the values of the positive G-onset rate $n$ of the same magnitude are reversed.

\section{Conclusions}

The purpose of the work is to present a way to solve the problem of correctly defining complex acceleration profiles that are recreated by a centrifuge motion simulator. The proposed solution, in the form of an inverse kinematic model of the centrifuge, indicates not only the exceeded limit values of the parameters, but also their changes over time. The simulation has revealed that the developed inverse kinematic model makes it possible to calculate the angular displacement, velocity and acceleration of the links, which is needed for the given linear acceleration of the simulator cabin. Simulation performed in Simulink proved the correctness of the presented expressions for angular displacement, velocity and acceleration of the centrifuge links. The presented algorithm achieved the predefined profile of absolute acceleration in the centrifuge cabin where the onset rate of the absolute acceleration is constant. The developed model of the inverse kinematics can be used for computer simulation of motion of the centrifuge simulator system. Through an overview of the behaviour of the model under various operating conditions, it is possible to predict how a real system will behave.

\section{References}

1. AMST-Systemtechnik GmbH, 2011, User manual Human Training Centrifuge HTC-07

2. BAillieul J., 1985, Kinematic programming alternatives for redundant manipulators, Proceedings of 1985 IEEE International Conference on Robotics and Automation, St. Louis, MO, USA, 722-728 
3. Buss S.R., Kim J.S., 2005, Selectively damped least squares for inverse kinematics, Journal of Graphics Tools, 10, 37-49

4. Chen Y.C., Repperger D.W., 1996, A study of the kinematics, dynamics and control algorithms for a centrifuge motion simulator, Mechatronics, 6, 829-852

5. Crosbie R.J., 1988, Dynamic Flight Simulator Control System, United States Patent, Number $4,751,662$

6. Dančuo Z., Rasuo B., Bengin A., Zelukovic V., 2018, Flight to Mars: Envelope simulation in a ground based high-performance human centrifuge, FME Transactions, 46, 1-9

7. Dančuo Z., Rasuo B., Vidaković J., Kvrgić V., Bućan M., 2013, On mechanics of a high-G human centrifuge, Proceedings in Applied Mathematics and Mechanics, 39-40

8. Dančuo Z., Vidaković J., Kvrgic V., Ferenc G., Lutovac M., 2012a, Modeling a human centrifuge as three-DoF robot manipulator, 2012 Mediterranean Conference on Embedded Computing, MECO, IEEE, 149-152 ISBN 9940943601

9. Dančuo Z., Zeluković V., Rašuo B., Dapić M., 2012b, High-G training profiles in a high performance human centrifuge, Scientific and Technical Review, 62, 64-69

10. Duuric A., Al Saidi R., Elmaraghy W., 2012, Dynamics solution of n-DOF global machinery model, Robotics and Computer-Integrated Manufacturing, 28, 621-630

11. Gherman B., Pisla D., Vaida C., Plitea N., 2012, Development of inverse dynamic model for a surgical hybrid parallel robot with equivalent lumped masses, Robotics and Computer-Integrated Manufacturing, 28, 402-415

12. Grotjahn M., Heimann B., Kuehn J., Grendel H., 2004, Dynamics of robots with parallel kinematic structure, The 11th World Congress in Mechanism and Machine Science, 1689-1693

13. Kvrgic V.M., Vidaković J., Lutovac M.M., Ferenc G.Z., Cvijanovic V.B., 2014, A control algorithm for a centrifuge motion simulator, Robotics and Computer-Integrated Manufacturing, 30, 399-412

14. Liwen G., Hui L.I.U., Meng F.U., 2015, Real-time motion planning algorithm for dynamic flight simulators (in Chinese), Tsinghua Science and Technology, 55, 709-715

15. NAKAmura Y., HanAfusa H., 1986, Inverse kinematic solutions with singularity robustness for robot manipulator control, Journal of Dynamic Systems Measurement and Control, 108, 163-171

16. Nearchou A.C., 1998, Solving the inverse kinematics problem of redundant robots operating in complex environments via a modified genetic algorithm, Mechanism and Machine Theory, 33, 273-292

17. Newman D.G., 2015, High G Flight: Physiological Effects and Countermeasures, 1st ed., Ashgate Publishing Ltd., Monash University, Australia, ISBN 9781472414571

18. Siciliano B., Sciavicco L., Villani L., Oriolo G., 2009, Robotics: Modelling, Planning and Control, Soft Computing, Springer, ISBN 9781846286414

19. Tejomurtula S., KaK S., 1999, Inverse kinematics in robotics using neural networks, Information Sciences (Ny), 116, 147-164

20. Truszczý́ski O., Kowalczuk K., 2012, The Polish centrifuge as a dynamic flight simulator. New application and ideas, Polish Journal of Aviation Medicine and Psychology, 18, 71-80

21. Tsai L., 1999, Robot Analysis: the Mechanics of Serial and Parallel Manipulators, 1st ed., John Wiley \& Sons, Inc., New York, NY, USA, ISBN 978-0-471-32593-2

22. Vidaković J., Ferenc G., Lutovac M., Kvrgic V., 2012, Development and implementation of an algorithm for calculating angular velocity of main arm of human centrifuge, 15th International Power Electronics and Motion Control Conference (EPE/PEMC), Novi Sad, Serbia, DS2a.17-1-DS2a.17-6 
23. Vidaković J., Lazarevic M., Kvrgic V.M., Dančuo Z., Lutovac M.M., 2013, Comparison of numerical simulation models for open loop flight simulations in the human centrifuge, Proceedings in Applied Mathematics and Mechanics, 485-486

24. WAmpler C.W., 1986, Manipulator inverse kinematic solutions based on vector formulations and damped least-squares methods, IEEE Transactions on Systems, Man, and Cybernetics, 16, 93-101

25. Wojtkowiak M., 1991, Human centrifuge training of men with lowered + Gz acceleration tolerance, Physiologist, 34, 80-82

26. Wolovich W.A., Elliott H., 1984, A computational technique for inverse kinematics, The 23rd IEEE Conference on Decision and Control, Las Vegas, Nevada, USA, 1359-1363

27. Wrigge S., 1981, Calculation of the Taylor series expansion coefficients of the Jacobian elliptic function $\operatorname{sn}(x, k)$, Mathematics of Computation, 36, 555-564

28. Wu J., Chen X., Li T., Wang L., 2013, Optimal design of a 2-DOF parallel manipulator with actuation redundancy considering kinematics and natural frequency, Robotics and Computer-Integrated Manufacturing, 29, 80-85

29. Wu J., Wang J., Wang L., Li T., 2009, Dynamics and control of a planar 3-DOF parallel manipulator with actuation redundancy, Mechanism and Machine Theory, 44, 835-849

30. Wu J., Wang J., You Z., 2010, An overview of dynamic parameter identification of robots, Robotics and Computer-Integrated Manufacturing, 26, 414-419

31. Zhaо Y., GAO F., 2009, Inverse dynamics of the 6-DOF out-parallel manipulator by means of the principle of virtual work, Robotica, 27, 259-268

Manuscript received January 14, 2018; accepted for print July 26, 2018 